\title{
Marked Hypercholesterolemia Caused by Mitotane Adjuvant Chemotherapy for Adrenocortical Carcinoma
}

Banu Sarer Yurekli, Nilufer Ozdemir Kutbay, Ilker Altun, Gokhan Ozgen

Ege University, Department of Endocrinology, Izmir, Turkey

\section{OBJECTIVES}

Mitotane (o,p'-DDD) has been used to treat adrenocortical carcinoma (ACC) for several decades. Mitotane is often given in adjuvant setting after surgical resection of $A C C$ and treatment usually lasts 2-3 years to reduce $A C C$ recurrence. The use of mitotane is associated with multiple adverse effects. We herein report a case of marked hypercholesterolemia in a man receiving mitotane as adjuvant chemotherapy for adrenocortical carcinoma.

\section{CASE}

A 64-year-old man was admitted to our Endocrinology Department with the laboratory findings of adrenal insufficiency. Mitotane was started as adjuvant chemotherapy for ACC by the Oncology department. With the usage of $4 \mathrm{gr}$ mitotane for 25 days, laboratory results were as follows: ACTH:298 pg/ml, cortisol:5.7 $\mu \mathrm{g} / \mathrm{dl}$, DHEAS:7.3 $\mu \mathrm{g} / \mathrm{dl}$. Hydrocortisone was started as 30 $\mathrm{mg} /$ day. As Potassium levels were normal with the hydrocortisone treatment, fludrocoritsone was not added to the treatment. Interestingly, the patient developed marked hyper-LDL cholesterolemia at a level of $236 \mathrm{mg} / \mathrm{dl}$ following introduction of mitotane. Before the mitotane treatment, LDL level of the patient was $139 \mathrm{mg} / \mathrm{dl}$. After mitotane treatment, total cholesterol was 370 $\mathrm{mg} / \mathrm{dl}$, triglyceride was $160 \mathrm{mg} / \mathrm{dl}$, HDL was $94 \mathrm{mg} / \mathrm{dl}$. TSH level of the patient was $1.7 \mu \mathrm{lU} / \mathrm{ml}$. There was no findings of hypogonadism. There was no medical history of primary familial and secondary hyperlipidemia. Low dose atorvastatin was started for hyperlipidemia.

\section{RESULTS}

In the literature, 21 Cushing's syndrome patients treated with mitotane exhibited increased cholesterol level (primarily LDL cholesterol) with an average of $68 \%$ increase. Mitotane may increase cholesterol by inhibiting the formation of the oxysterol. It may decrease cholesterol catabolism by inhibiting the production of cholesterol oxidase. Mitotane may be able to stimulate the activity of HMG-CoAreductase strongly enough to significantly increase LDL-cholesterol synthesis.

\section{CONCLUSIONS}

TextWe recommend careful monitoring of serum cholesterol level following the introduction of mitotane chemotherapy. 